\title{
Efectividad de los Indicadores de Gestión en la Recaudación de Rentas de las Municipalidades de Lima-Metropolitana 2013-2015
}

\author{
Effectiveness of the Management Indicators in \\ Raising Revenue Municipalities of Lima Metropolitana 2013-2015
}

Pedro Jaime Valencia Pomareda* hervalpo@gmail.com

[RECEPCIÓN: AGOSTO 2016 / CONFORMIDAD: OCTUBRE 2016]

\begin{abstract}
RESUMEN
De acuerdo a los artículos 97 y 98 de la Ley Orgánica de Municipalidades № 27972, se instaura la coordinación entre los gobiernos locales, estableciéndose un plan piloto que cuenta con un Tablero de Mando-BSC, cuyos indicadores son compartidos por los gobiernos locales de Lima Metropolitana, con resultados no muy satisfactorios. Con el fin de salvar esta dificultad, se ha emprendido un plan piloto de gestión estratégica, con el Tablero de Mando-BSC, que interconecte las perspectivas, indicadores, metas e iniciativas. Habiendo revisado este tablero, se deduce que probablemente, entre otras variables, la debilidad se encuentre en los indicadores de gestión, por lo que hasta la fecha no se logran los objetivos deseados.
\end{abstract}

Palabras clave: Tablero de mando integral; gestión municipal; indicadores de eficiencia y eficacia de la gestión

\section{ABSTRACT}

According to Articles 97 and 98 of the Organic Law of Municipalities $\mathrm{N}^{\circ} 27972$, coordination between local governments is established, establishing a pilot plan that features a Dashboard-BSC, whose indicators are shared by local governments Lima, with not very satisfactory results. In order to overcome this difficulty has undertaken a pilot strategic management plan, with the Balanced Scorecard-BSC, which interconnects prospects, indicators, targets and initiatives. Having reviewed this board follows that probably, among other variables, the weakness is in the management indicators, so far not achieved the desired objectives.

Keywords: balanced scorecard; municipal management; indicators of efficiency and management effectiveness.

Doctor en Ciencias Administrativas-UNMSM. Magíster en Administración, con mención en Gestión Empresarial-UNMSM. Experto en Administración Pública. Licenciado en Administración. Docente de pregrado y posgrado. 


\section{INTRODUCCIÓN}

Teniendo diseñado un Cuadro de Mando Integral-BSC piloto, para las áreas de captación de fondos, queremos conocer y verificar si sus indicadores son eficaces y eficientes, que permitan conseguir que los presupuestos programados para impuestos prediales y arbitrios se asemejen a los recursos captados, con menores gastos, lo que favorecería la efectividad de la gestión municipal.

¿Cómo se relaciona el Tablero de Mando-BSC con el nivel de eficacia y eficiencia de los indicadores de recaudación del impuesto predial y arbitrios en la gestión de los gobiernos locales de Lima Metropolitana 2013-2015?

- ¿Cómo se relaciona el Tablero de Mando BSC con el nivel de eficacia en los indicadores de recaudación por impuesto predial en la gestión de los gobiernos locales de Lima Metropolitana 2013-2015?

- ¿Cómo se relaciona el Tablero de MandoBSC con el nivel de eficiencia en los indicadores de recaudación por impuesto predial en la gestión de los gobiernos locales de Lima Metropolitana 2013-2015?

- ¿Cómo se relaciona el Tablero de MandoBSC con nivel de eficacia en los indicadores de recaudación por arbitrios, en la gestión de los gobiernos locales de Lima Metropolitana 2013-2015?

El objetivo de la investigación es: (1) Determinar cómo se relaciona el Tablero de Mando-BSC con el nivel de eficacia y eficiencia de los indicadores, para la recaudación del impuesto predial y arbitrios en los gobiernos locales de Lima Metropolitana 2013-2015. (2) Determinar cómo se relaciona el Tablero de Mando-BSC con el nivel de eficacia en los indicadores de recaudación del impuesto predial en los gobiernos locales de Lima Metropolitana 2013-2015. (3) Determinar cómo se relaciona el Tablero de Mando-BSC con el nivel de eficiencia en los indicadores de recaudación del impuesto predial en los gobiernos locales de Lima Metropolitana 2013-2015. (4) Determinar cómo se relaciona el Tablero de Mando-BSC con el nivel de eficacia en los indicadores de recaudación por arbitrios en los gobiernos locales de Lima Metropolitana 2013-2015. (5) Determinar cómo se relaciona el Tablero de Mando-BSC con el nivel de eficiencia en los indicadores de recau- dación de arbitrios en los gobiernos locales de Lima Metropolitana 2013-2015.

\section{SISTEMA DE HIPÓTESIS MÉTODO \\ TABLERO DE MANDO BSC}

Según Salguero (2011), si se mejora el aprendizaje y se desarrolla más la tecnología en una organización mediante el control y el cumplimiento de los indicadores planteados, entonces se cuenta con lo necesario para mejorar los indicadores que se planteen en los procesos internos de la organización.

Si se mejoran los procesos internos, entonces se puede mejorar la orientación a los usuarios; si se mejora la relación con los usuarios-clientes, por consiguiente, se mejoran los resultados financieros de una organización.

\section{PERSPECTIVAS DEL BALANCED SCORE CARD}

Se presentan los conceptos de las cuatro perspectivas planteados por Kaplan y Norton (2000). Es importante mencionar que las empresas no solo deben limitarse a la utilización de las cuatro perspectivas: las financieras, las perspectivas del cliente, las perspectivas de procesos internos y las perspectivas de aprendizaje y crecimiento, pudiendo agregarse las que puedan adaptarse al negocio de las mismas, lo que sí no es usual contar con más de seis.

\section{ESQUEMA DE MEDICIONES}

Según Mejía, estos indicadores son los siguientes:

Eficacia: Grado en que se logran los objetivos y metas de un plan, es decir, cuando el resultado esperado se alcanzó.

La eficacia consiste en lograr objetivos mediante la concentración de los esfuerzos de una entidad en las actividades y los procesos que deben llevarse a cabo para el cumplimiento de los objetivos formulados.

Eficiencia: Es el logro de un objetivo al menor costo posible. En este caso, estamos buscando un empleo óptimo de los recursos disponibles para lograr los objetivos deseados.

Efectividad: Este concepto involucra la eficacia y la eficiencia, es decir, el logro de los resultados 
programados en el tiempo y con los costos más razonables posibles. Supone hacer lo correcto con gran exactitud y sin ningún desperdicio de tiempo y dinero.

El tipo de investigación será no experimental, utilizando el método correlacional.

\section{RESULTADOS}

Población: Está formada por los 43 gobiernos distritales de la provincia de Lima Metropolitana.

Tamaño de la muestra: Está constituida por 40 gobiernos locales distritales.

Selección de muestra: Fue de tipo aleatoria simple autoponderada por conveniencia, para la recaudación de impuesto predial y arbitrios entre los gobiernos locales de Lima Metropolitana.

\section{RESULTADOS DE LA EFECTIVIDAD DEL TABLERO DE MANDO}

Para el análisis del cumplimiento de los indicadores planteados en las cuatro perspectivas del tablero de mando, se ha utilizado la escala de evaluación porcentual siguiente:

\begin{tabular}{|c|c|}
\hline Rango cuantitativo & Rango cualitativo \\
\hline $61-91 \%$ & Alta (3) \\
\hline $60-41 \%$ & Regular (2) \\
\hline $40-20 \%$ & Baja (1) \\
\hline
\end{tabular}

\section{CARACTERIZACIÓN DEL TABLERO DE MANDO BSC}

El cuadro uno presenta los puntajes promedios y la desviación típica de los indicadores del Tablero de Mando BSC. De una puntuación de 1 a 3, la media más alta $(M=2.28)$ se dio en el ítem 8 , Indicadores de satisfacción de usuarios, y la más baja $(M=1.75)$ en el ítem 13 , Indicadores de ingreso por recuperación por ejecución coactiva. Otros reactivos obtuvieron medias por encima del puntaje medio de 2, evidenciando niveles altos: 1) Indicadores de desempeño personal $(M=2.05)$, 9) Indicadores de incremento de contribuyentes $(M=2.08), 10)$ Indicadores de participación en eventos de cultura tributaria ( $M=2.03), 11)$ Indicadores de cumplimiento tributario $(\mathrm{M}=2.00)$ y 12) Indicadores de acogimiento de amnistía tributaria $(\mathrm{M}=2.03)$. Con puntuaciones promedio más bajos están los ítems: 2) Indicadores de sistemas de información $(\mathrm{M}=1.88), 3)$ Indicadores de innovación para generar culturas tributarias $(M=1.85), 4)$ Indicadores para incorporar a la formalidad tributaria $(\mathrm{M}=1.88), 5)$ Indicadores para la solución de reclamos $(M=1.90), 6)$ Indicadores para la notificación programada a contribuyentes $(\mathrm{M}=1.83), 7)$ Indicadores de declaraciones juradas atendidas $(\mathrm{M}=1.93)$ (Ver figura uno). La mayor dispersión de los datos se dio en el ítem 10) Indicadores de participación en eventos de cultura tributaria $(\mathrm{DT}=.800)$ y la menor dispersión en el ítem 1) Indicadores de desempeño personal $(\mathrm{DT}=.677)$.

Cuadro $\mathrm{N}^{\mathrm{O}}$ 1. Media y DT de los indicadores del Tablero de Mando-BSC

\begin{tabular}{|c|c|c|c|c|}
\hline $\begin{array}{l}\text { INDICADORES TABLERO DE } \\
\text { MANDO BSC }\end{array}$ & Mín & Máx & Media & D.T. \\
\hline $\begin{array}{l}\text { 1) Indicadores de desempeño } \\
\text { personal }\end{array}$ & 1 & 3 & 2.05 & .677 \\
\hline $\begin{array}{l}\text { 2) Indicadores de sistemas de } \\
\text { información }\end{array}$ & 1 & 3 & 1.88 & .686 \\
\hline $\begin{array}{l}\text { 3) Indicadores de innovación para } \\
\text { generar culturas tributarias }\end{array}$ & 1 & 3 & 1.85 & .770 \\
\hline $\begin{array}{l}\text { 4) Indicadores para incorporar a la } \\
\text { formalidad tributaria }\end{array}$ & 1 & 3 & 1.88 & .723 \\
\hline $\begin{array}{l}\text { 5) Indicadores para la solución de } \\
\text { reclamos }\end{array}$ & 1 & 3 & 1.90 & .778 \\
\hline $\begin{array}{l}\text { 6) Indicadores para la notificación } \\
\text { programada a contribuyentes }\end{array}$ & 1 & 3 & 1.83 & .747 \\
\hline $\begin{array}{l}\text { 7) Indicadores de declaraciones } \\
\text { juradas atendidas }\end{array}$ & 1 & 3 & 1.93 & .797 \\
\hline $\begin{array}{l}\text { 8) Indicadores de satisfacción de } \\
\text { usuarios }\end{array}$ & 1 & 3 & 2.28 & .784 \\
\hline $\begin{array}{l}\text { 9) Indicadores de incremento de } \\
\text { contribuyentes }\end{array}$ & 1 & 3 & 2.08 & .764 \\
\hline $\begin{array}{l}\text { 10) Indicadores de participación } \\
\text { en eventos de cultura tributaria }\end{array}$ & 1 & 3 & 2.03 & .800 \\
\hline $\begin{array}{l}\text { 11) Indicadores de cumplimiento } \\
\text { tributario }\end{array}$ & 1 & 3 & 2.00 & .784 \\
\hline $\begin{array}{l}\text { 12) Indicadores de acogimiento de } \\
\text { amnistía tributaria }\end{array}$ & 1 & 3 & 2.13 & .757 \\
\hline $\begin{array}{l}\text { 13) Indicadores de ingreso por re- } \\
\text { cuperación por ejecución coactiva }\end{array}$ & 1 & 3 & 1.75 & .707 \\
\hline $\begin{array}{l}\text { 14) Indicadores en la generación } \\
\text { de disponibilidad financiera }\end{array}$ & 1 & 3 & 1.90 & .744 \\
\hline TABLERO DE MANDO-BSC & 1 & 3 & 1.93 & .730 \\
\hline
\end{tabular}

TOTAL DE INDICADORES DEL TABLERO DE MANDOBSC

\section{INTERPRETACIÓN}

En el cuadro 2, se observa que el $47.5 \%$ de los casos señalan que los indicadores del Tablero de 
Mando BSC se ubican en el nivel regular de eficiencia, el $22.5 \%$ en el nivel alto y el $30.0 \%$ en el nivel bajo. En el histograma de la curva normal de las frecuencias nos muestra una media de 1.93, con una desviación típica de 730, de un total de 40 .

Cuadro $N^{\circ} 2$. Frecuencia y porcentaje del total de indicadores del Tablero de Mando BSC, según niveles TOTAL DE INDICADORES DEL TABLERO DE MANDOBSC

\begin{tabular}{|c|c|c|c|}
\hline NIVELES & Frecuencia & Porcentaje & $\begin{array}{c}\text { Porcentaje } \\
\text { acumulado }\end{array}$ \\
\hline Bajo & 12 & 30.0 & 30.0 \\
\hline Regular & 19 & 47.5 & 77.5 \\
\hline Alto & 9 & 22.5 & 100.0 \\
\hline Total & 40 & 100.0 & \\
\hline
\end{tabular}

la eficacia y la eficiencia en los resultados de recaudación de predios y arbitrios.

Caracterización de los indicadores de eficiencia y eficacia en la recaudación de predios y arbitrios

En el cuadro 3, se presentan los puntajes promedios y la desviación típica y la escala de los indicadores de eficiencia y eficacia en la recaudación de predios y arbitrios.

De una puntuación de 1 a 3 la media más alta se dio en el ítem 4) Indicadores de eficiencia en los resultados de recaudación de arbitrios $(\mathrm{M}=2.08)$ y las más bajas con medias de 1.95 en los ítems 1) Indicadores de eficacia en los resultados de recaudación de predios, 2) Indicadores de eficiencia en los resultados de recaudación de predios y 3) Indicadores de eficacia en los resultados de recaudación de arbitrios.

La mayor dispersión de los datos se dio en el ítem 4) Indicadores de eficiencia en los resultados de recaudación de arbitrios y la menor dispersión en el ítem 3) Indicadores de eficacia en los resultados de recaudación de arbitrios.

En síntesis, la eficiencia y la eficacia en la recaudación de predios y arbitrios es de nivel medio, con mejor eficiencia en los resultados de recaudación de predios y la mayoría de ítems presentan dispersión moderada.
Cuadro No 3. Media y D.T. de los indicadores de Eficiencia y Eficacia en la recaudación de predios y arbitrios

\begin{tabular}{|l|c|c|c|c|c|}
\hline $\begin{array}{c}\text { EFICIENCIA Y EFICA- } \\
\text { CIA EN PREDIOS Y } \\
\text { ARBITRIOS }\end{array}$ & N & Mínimo & Máximo & Media & D.T. \\
\hline $\begin{array}{l}\text { 1)Indicadores de efica- } \\
\text { cia en los resultados de } \\
\text { recaudación de predios }\end{array}$ & 40 & 1 & 3 & 1.95 & .749 \\
\hline $\begin{array}{l}\text { 2)Indicadores de efi- } \\
\text { ciencia en los resulta- } \\
\text { dos de recaudación de } \\
\text { predios }\end{array}$ & 40 & 1 & 3 & 1.95 & .749 \\
\hline $\begin{array}{l}3 \text { )Indicadores de efica- } \\
\text { cia en los resultados de } \\
\text { recaudación de arbitrios }\end{array}$ & 40 & 1 & 3 & 1.95 & .639 \\
\hline $\begin{array}{l}\text { 4)Indicadores de efi- } \\
\text { ciencia en los resulta- } \\
\text { dos de recaudación de } \\
\text { arbitrios }\end{array}$ & 40 & 1 & 3 & 2.08 & .829 \\
\hline $\begin{array}{l}\text { TOTAL EFICIENCIA Y } \\
\text { EFICACIA }\end{array}$ & 40 & 1 & 3 & 1.98 & .648 \\
\hline
\end{tabular}

\section{PRUEBAS DE HIPÓTESIS}

\section{HIPÓTESIS GENERAL (HG)}

\section{$1{ }^{\circ}$ FORMULACIÓN DE LA HIPÓTESIS DE TRABAJO}

H1: El tablero de mando-BSC es de alta efectividad para alcanzar indicadores de eficacia y eficiencia en los resultados de recaudación del impuesto predial y arbitrios para la gestión municipal de los gobiernos locales de Lima Metropolitana.

Ho: El total de indicadores del nivel de efectividad de la aplicación del Tablero de Mando-BSC no se relaciona significativamente con los niveles de los indicadores de eficiencia y eficacia en los resultados de recaudación del impuesto predial y de arbitrios para la gestión municipal de los gobiernos locales.

\section{$2^{\circ}$ NIVELES DE SIGNIFICACIÓN}

$$
\alpha=0.05 \text { (con 95\% de confianza) }
$$

\section{$3^{\circ}$ ESTADÍSTICOS DE PRUEBA}

Para hallar la correlación entre las variables de estudio, se aplicará la Tau b de Kendall: 
S

$\mathrm{Tb}=$

$\sqrt{ } 1 / 2 \mathrm{~N}(\mathrm{~N}-1)-\mathrm{Tx} \sqrt{ } 1 / 2 \mathrm{~N}(\mathrm{~N}-1)-\mathrm{Ty}$

Donde: $\mathrm{S}=$ Suma de rangos de las variables $\mathrm{X}$ e $\mathrm{Y}$

$$
\begin{aligned}
& \mathrm{Tx},=1 / 2 \sum \mathrm{t}(\mathrm{t}-1) \\
& \mathrm{Ty}=1 / 2 \sum \mathrm{t}(\mathrm{t}-1) \\
& \mathrm{T}=\text { Número de empates en la variable } \mathrm{X} \text { e } \mathrm{Y} \\
& \mathrm{N}=\text { Número de casos }
\end{aligned}
$$

Estadístico de prueba de significación para la $r$ hallada es la prueba $t$

Donde:

En términos generales, diremos que:

Si el p valor de la r (Correlación de Tau-b) < 0.05 la asociación se cumple.

Si el p valor de la $r$ (Correlación de Tau-b) $>$ 0.05 la asociación no se cumple.

\section{$4^{\circ}$ COMPARAR}

El valor tabular o teórico de la t de Student al 95\% de confianza, con 38 grados de libertad y para un contraste bilateral o de dos colas, es 2.021, con lo cual la hipótesis nula Ho será rechazada si el valor calculado o muestra de la prueba t es menor que -2.021 o es mayor que 2.021; en caso contrario, será aceptada. En términos del p valor, la hipótesis nula (Ho) será rechazada si el p valor es menor que el nivel de significación usual de 0.05 . Las zonas de aceptación y de rechazo de la hipótesis nula Ho se ilustra en la figura 1.

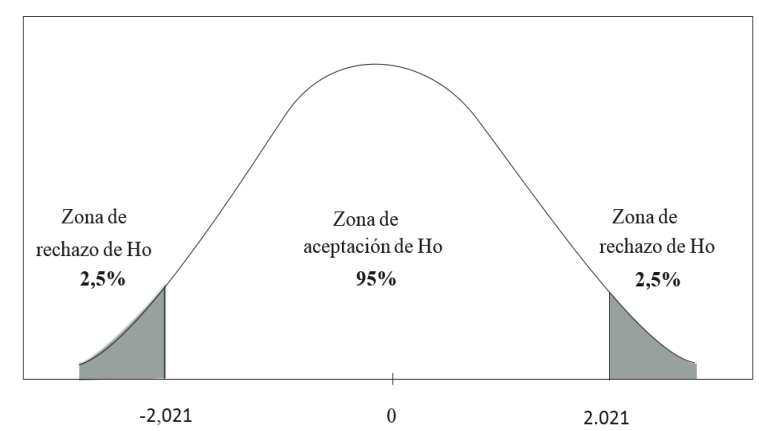

Figura $N^{\circ} 1$. Zona de aceptación o rechazo de la $\mathrm{H}_{\mathrm{o}}$
En el cuadro 4, teniendo en cuenta que las dos variables de estudio se miden con una escala ordinal, se ve que el coeficiente de correlación Tb de Kendall hallado es de 0.432, de donde, reemplazando en la prueba t, se obtiene que el valor calculado de $t$ es 2.615 y reporta un p valor de 0.009 . Comparando los valores calculados (tc $=2,615)$ y teórico $(\mathrm{tt}=2.021)$ de la $\mathrm{t}$ de Student se aprecia que el primero es mayor que el segundo, por lo que se rechaza la hipótesis nula (Ho) a favor de la hipótesis alternativa (Ha). Por otra parte, el p valor (0) es menor que el nivel usual de significación de 0,05 (incluso con el nivel habitual de 0,01$)$, corroborando la decisión anterior. Al 95\% de probabilidad, se demuestra que: "Existe correlación entre los niveles de efectividad de la aplicación del Tablero de Mando y los niveles de eficiencia y eficacia en la recaudación de predios y arbitrios", con lo cual se comprueba la hipótesis general de investigación.

\begin{tabular}{|c|c|c|c|c|}
\hline \multicolumn{2}{|c|}{ ESCALAS ESTADÍSTICAS } & Valor & T aprox(a) & Sig. aprox \\
\hline $\begin{array}{c}\text { Nominal por } \\
\text { nominal }\end{array}$ & $\begin{array}{l}\text { Coeficiente de } \\
\text { contingencia }\end{array}$ & .583 & & .000 \\
\hline \multirow[t]{2}{*}{$\begin{array}{l}\text { Ordinal por } \\
\text { ordinal }\end{array}$} & $\begin{array}{l}\text { Tau-b de } \\
\text { Kendall }\end{array}$ & .432 & 2.615 & .009 \\
\hline & $\begin{array}{l}\text { Correlación } \\
\text { de Spearman }\end{array}$ & .454 & 3.143 & $.003(b)$ \\
\hline $\begin{array}{l}\text { Intervalo por } \\
\text { intervalo }\end{array}$ & $\mathrm{R}$ de Pearson & .454 & 3.142 & $.003(b)$ \\
\hline
\end{tabular}

Cuadro No4. Correlación de Kendall del Tablero de Mando-BSC y la eficiencia y la eficacia en recaudación de predios y arbitrios

- Empleando el error típico asintótico basado en la hipótesis nula.

- Basada en la aproximación normal.

\section{Conclusión}

Se concluye que: Existe correlación directa, media y significativa entre el nivel de eficacia y eficiencia entre los indicadores de recaudación de predios y arbitrios y el Tablero de Mando-BSC de los gobiernos locales de Lima Metropolitana. Es decir, a mayor nivel de efectividad del Tablero de 
Mando-BSC, mayor nivel de eficacia y eficiencia en la recaudación de predios y arbitrios.

\section{HIPÓTESIS ESPECIIFICAS}

\section{HIPÓTESIS ESPECÍFICA 1 (HE1)}

\section{$1^{\circ}$ FORMULACIÓN DE HIPÓTESIS DE TRABAJO H1}

Cuadro $\mathrm{N}^{0}$ 5. Correlación de Kendall del Tablero de Mando-BSC y los indicadores de eficacia en la recaudación de predios

\begin{tabular}{|c|c|c|c|c|}
\hline \multicolumn{2}{|c|}{ ESCALAS ESTADÍSTICAS } & Valor & T aprox.(a) & $\begin{array}{c}\text { Sig. } \\
\text { Aprox }\end{array}$ \\
\hline $\begin{array}{c}\text { Nominal por } \\
\text { nominal }\end{array}$ & $\begin{array}{c}\text { Coeficiente de } \\
\text { contingencia }\end{array}$ & .410 & .088 \\
\hline $\begin{array}{c}\text { Ordinal por } \\
\text { ordinal }\end{array}$ & $\begin{array}{c}\text { Tau-b de } \\
\text { Kendall }\end{array}$ & .381 & 2.956 & .003 \\
\hline & $\begin{array}{c}\text { Correlación } \\
\text { de Spearman }\end{array}$ & .419 & 2.848 & $.007(\mathrm{~b})$ \\
\hline
\end{tabular}

- Empleando el error típico asintótico basado en la hipótesis nula.

- Basada en la aproximación normal.

\section{CONCLUSIONES}

Se concluye que: Existe correlación directa, media y significativa, entre el nivel de eficacia entre los indicadores de recaudación de predios y el Tablero de Mando-BSC de los gobiernos locales de Lima Metropolitana. Es decir, a mayor nivel de efectividad en el Tablero de Mando BSC, mayor nivel de eficacia en la recaudación de predios.

\section{HIPÓTESIS ESPECÍFICA 2 (HE2)}

Cuadro $N^{\circ} 6$. Correlación de Kendall del Tablero de Mando-BSC y la eficiencia en recaudación de predios.

\begin{tabular}{|c|c|c|c|c|}
\hline \multicolumn{2}{|c|}{ ESCALA ESTADÍSTICA } & Valor & $\begin{array}{c}\text { T } \\
\text { aprox(a) }\end{array}$ & $\begin{array}{c}\text { Sig. } \\
\text { aprox }\end{array}$ \\
\hline $\begin{array}{c}\text { Nominal por } \\
\text { nominal }\end{array}$ & $\begin{array}{c}\text { Coeficiente de } \\
\text { contingencia }\end{array}$ & .348 & .238 \\
\hline $\begin{array}{c}\text { Ordinal por } \\
\text { ordinal }\end{array}$ & $\begin{array}{c}\text { Tau-b de } \\
\text { Kendall }\end{array}$ & .332 & 2.624 & .009 \\
\hline & $\begin{array}{c}\text { Correlación de } \\
\text { Spearman }\end{array}$ & .369 & 2.446 & $.019(\mathrm{~b})$ \\
\hline
\end{tabular}

Empleando el error típico asintótico basado en la hipótesis nula.

- Basada en la aproximación normal.

\section{CONCLUSIÓN}

Se concluye que: Existe correlación directa, media y significativa, entre los niveles de efectividad de la aplicación del Tablero de Mando-BSC y los niveles de eficiencia en la recaudación de predios en los gobiernos locales. Es decir, a mayor nivel de efectividad en el Tablero de Mando BSC, mayor nivel de eficiencia en la recaudación de predios.

\section{HIPÓTESIS ESPECÍFICA 3 (HE3)}

Cuadro $\mathrm{N}^{0} 7$. Correlación de Kendall del Tablero de Mando-BSC y la eficacia en recaudación de arbitrios.

\begin{tabular}{|c|c|c|c|c|}
\hline \multicolumn{2}{|c|}{ ESCALAS ESTADÍSTICAS } & Valor & $\begin{array}{c}\text { T aprox. } \\
\text { (a) }\end{array}$ & $\begin{array}{c}\text { Sig. } \\
\text { Aprox. }\end{array}$ \\
\hline $\begin{array}{c}\text { Nominal por } \\
\text { nominal }\end{array}$ & $\begin{array}{c}\text { Coeficiente de } \\
\text { contingencia }\end{array}$ & .355 & .218 \\
\hline $\begin{array}{c}\text { Ordinal por } \\
\text { ordinal }\end{array}$ & $\begin{array}{c}\text { Tau-b de Ken- } \\
\text { dall }\end{array}$ & .145 & .869 & .385 \\
\hline
\end{tabular}

- Empleando el error típico asintótico basado en la hipótesis nula.

- Basada en la aproximación normal.

\section{CONCLUSIÓN}

Se concluye que: No existe correlación significativa entre los niveles de efectividad de la aplicación del Tablero de Mando y los niveles de eficacia en la recaudación de arbitrios en el municipio de San Borja. Es decir, el nivel efectividad en el Tablero de Mando BSC no se asocia al nivel de eficacia en la recaudación de arbitrios.

\section{HIPÓTESIS ESPECÍFICA 4 (HE4)}

Cuadro No8. Correlación de Kendall del Tablero de Mando-BSC y la eficiencia en recaudación de arbitrios

\begin{tabular}{|c|c|c|c|c|}
\hline \multicolumn{2}{|c|}{ ESCALAS ESTADÍSTICAS } & Valor & T aprox.(a) & $\begin{array}{c}\text { Sig. } \\
\text { Aprox. }\end{array}$ \\
\hline $\begin{array}{c}\text { Nominal por } \\
\text { nominal }\end{array}$ & $\begin{array}{c}\text { Coeficiente de } \\
\text { contingencia }\end{array}$ & .400 & .107 \\
\hline $\begin{array}{c}\text { Ordinal por } \\
\text { ordinal }\end{array}$ & $\begin{array}{c}\text { Tau-b de Ken- } \\
\text { dall }\end{array}$ & .197 & 1.274 & .203 \\
\hline
\end{tabular}

- Empleando el error típico asintótico basado en la hipótesis nula.

- Basada en la aproximación normal. 


\section{CONCLUSIONES}

Se concluye que: No existe correlación entre los niveles de efectividad de la aplicación del tablero de mando y los niveles de eficiencia en la recaudación de arbitrios en los gobiernos locales. Es decir, el nivel de efectividad del Tablero de Mando-BSC no se asocia al nivel de efectividad en la recaudación de arbitrio.

\section{DISCUSIÓN}

Estos resultados y su análisis nos llevan a proponer lo que otros estudios ya plantearon. La utilización de nuevos indicadores, una vez lograda su excelencia, o la mejora de los ya existentes, pretendiendo la mejora continua dentro de un ambiente de cambio y de aprender a aprender (Landeros, 2007 y Presas, 2007). Mejorar la perspectiva financiera, cuando no se cumple las obligaciones financieras inmediatas (Romero, 2005).

En el aspecto tecnológico, recomiendan que se deba configurar el cuadro de mando en cada área funcional, y en cada nivel de responsabilidad, de manera que albergue siempre la información mínima, necesaria y suficiente para poder extraer conclusiones y tomar decisiones acertadas. Finalmente, proponen un método singular, ventajoso y eficaz de construcción, ejecución y control del Balanced Score Card: Los tejidos de metas, restricciones y estrategias generan el tejido de indicadores, el que a su vez origina el tejido de responsables, sustento del ajuste organizacional. Más que motivar solamente la exploración de indicadores diferentes, el autor plantea que las mediciones sean radicalmente distintas.

Los resultados del nivel de indicadores de eficiencia y eficacia hallado en la recaudación del impuesto predial y de arbitrios para la gestión municipal de los gobiernos locales estudiados, indican un nivel medio o regular en más de la mitad de los casos estudiados y una puntuación media cercana a dos puntos, es decir que como eficiencia las cosas se están haciendo bien en un nivel regular y como eficacia se están haciendo las cosas debidas de manera regular (Salgueiro, 2001).

Al ver los resultados específicos en la efectividad en la recaudación de tributos, observamos que la puntuación media se acerca a dos y es igual en los casos de eficacia en predios, eficiencia en predios y eficacia en arbitrios. La eficiencia en los resultados de recaudación en arbitrios con una puntuación algo superior al puntaje medio de dos indica que el personal está consumiendo muchos recursos y obtiene bajos resultados en la recaudación de arbitrios.

Los resultados de las correlaciones halladas verifican la hipótesis general y las hipótesis específicas uno y dos, de que la variable niveles de efectividad de la aplicación del Tablero de Mando BSC se asocia, de manera directa y a un nivel moderado y bajo con la variable niveles de eficiencia y eficacia en los resultados de recaudación de predios en los municipios limeños.

Al no hallarse correlación entre los niveles del tablero de mando con los niveles de eficacia y eficiencia en la recaudación de arbitrios, podría explicarse por el elevado índice de morosidad en los pagos de este tributo en los contribuyentes del municipio, dado que el ser humano da más importancia a la propiedad individual (tributo en predios) que al cumplimiento de deudas por servicio público (tributo de arbitrios), que es parte de nuestra cultura tributaria.

\section{CONCLUSIONES}

De las pruebas de hipótesis y la discusión realizadas de los resultados estadísticamente se ha llegado a lo siguiente:

1. Existe correlación directa, media y significativa entre los niveles de efectividad de la aplicación del tablero de mando y los niveles de eficiencia y eficacia en la recaudación de predios y arbitrios en los gobiernos locales. Es decir, a mayor nivel de efectividad en el Tablero de Mando BSC, mayor nivel de eficiencia y eficacia en la recaudación de predios y arbitrios.

2. Existe correlación directa, media y significativa, entre los niveles de efectividad de la aplicación del tablero de mando y los niveles de eficiencia en la recaudación de predios en los gobiernos locales. Es decir, a mayor nivel efectividad en el Tablero de Mando BSC, mayor nivel de eficiencia en la recaudación de predios.

3. Existe correlación directa, media y significativa entre los niveles de efectividad de la aplicación del tablero de mando y los 
niveles de eficacia en la recaudación de predios en los gobiernos locales. Es decir, a mayor nivel efectividad en el Tablero de Mando BSC, mayor nivel de eficacia en la recaudación de predios.

4. Se concluye que: No existe correlación entre los niveles de efectividad de la aplicación del tablero de mando y los niveles de eficacia en la recaudación de arbitrios en los gobiernos locales. Es decir, el nivel efectividad en el Tablero de Mando BSC no se asocia al nivel de eficacia en la recaudación de arbitrios.

5. Se concluye que: No existe correlación entre los niveles de efectividad de la aplicación del tablero de mando y los niveles de eficiencia en la recaudación de arbitrios en los gobiernos locales. Es decir, el nivel efectividad en el Tablero de Mando BSC no se asocia al nivel de eficiencia en la recaudación de arbitrios.

\section{REFERENCIAS BIBLIOGRÁFICAS}

Hernández Sampieri Carlos, Fernández Collado Pilar, Baptista Lucio. (2008). Metodología de la investigación.

Kaplan, Robert y Norton, David (2000). El cuadro de mando integral. Barcelona: Gestión 2000.

Kaplan, Robert y Norton, David. (2004). Mapas estratégicos: Convirtiendo activos intangibles en resultados tangibles. Barcelona: Gestión 2000.

Salgueiro, A. (2011). Indicadores de Gestión y Cuadro de Mando. Madrid: Días de Santos S.A.

Kaplan, Robert., \& Norton, David. (2005). El Balanced score card: Mediciones que impulsan el desempeño. Harvard Deusto Business Review, 83(7), 102-110.

López, Alfonso (2004). Los sistemas de información basados en la estrategia. Finanzas \& Contabilidad, (46), 18-27. 